\title{
Editorial
}

\section{The Issues Related to Hemodialysis Catheter Lock Prophylaxis with Recombinant Tissue Plasminogen Activator}

\author{
Anil K. Saxena ${ }^{*}, 1$, Kenneth Abreo $^{2}$ and Tushar Vachharajani ${ }^{3}$ \\ ${ }^{I}$ Al-Rahba hospital- Johns Hopkins Medicine International, Abu Dhabi, United Arab Emirates (UAE) \\ ${ }^{2}$ LSU Health Sciences Center, Shreveport, LA 71130, USA \\ ${ }^{3}$ Wake Forest University School of Medicine, Medical Center Boulevard, Winston-Salem, NC 27157, USA
}

Arteriovenous fistulas (AVF) are the most preferred and promoted form of dialysis access due to their lowest complication rates. However, the changing demographic profile of end- stage renal disease (ESRD) with an increasing elderly and diabetic population having relatively poor vasculature, relative lack of prudent pre-ESRD planning for AV fistula creation, late referrals, and comparatively lower fistula maturation rates, are essentially the factors accountable for our continued dependence upon central venous catheters (CVCs) [1-3]. Moreover, CVCs remain the only means to provide instant access to the circulation during emergency situations arising from the unpredictable course of chronic kidney disease.

Virchow's described three broad categories of factors that contribute to thrombus formation -hypercoagulability, stasis and vascular endothelial injury. The placement of this relatively large foreign body traumatizes the host vessel and significantly alters hemodynamics fulfilling Virchow's triad for thrombus formation and provides a nidus for bacterial entry into the circulation. However, catheter malfunction limits the life span of CVCs. Inadequate blood flow and catheter-related bloodstream infections (CRBSI) are common causes of recurrent use of thrombolytic therapy, antibiotics and often result in catheter removal. Generally, standard heparin $(5000 \mathrm{U} / \mathrm{mL})$ is used to lock the CVCs to maintain catheter patency during the interdialytic period. Locking with heparin however, does not prevent bacterial colonization and subsequent development of CRBSI. Moreover, in vitro studies have shown that heparin may induce biofilm formation [4].

Replacing heparin with recombinant tissue plasminogen activator (rt-PA) once a week, as a dialysis catheter locking solution significantly reduced the incidence of catheter malfunction and CRBSI in a well conducted multicenter, double-blind, randomized controlled trial from Canada by Hemmelgarn and colleagues [5]. The authors were able to decrease the rates of catheter malfunction by $50 \%$ and

*Address correspondence to this author at the Nephrology Division, Department of Medicine, Al-Rahba hospital- Johns Hopkins Medicine International, PO Box 34555, Health Authority Abu Dhabi, Abu Dhabi, United Arab Emirates (UAE); Tel: (+971) 506370180; Fax: (+971) 25064000; E-mail: dranil_31982@yahoo.com
CRBSI by two-thirds, with the use of rt-PA once a week at a dose $1 \mathrm{mg}$ per lumen. Patients $(\mathrm{n}=115)$ were randomly assigned to a catheter-locking group with heparin $(5,000$ $\mathrm{U} / \mathrm{mL}$ ) three times a week or with rt-PA (1 mg per lumen) substituted for heparin once a week and heparin used the remaining two times $(\mathrm{n}=110)$. Mean follow-up period after randomization was six months with treatment assignments concealed from patients, investigators, and trial staff. The primary outcome was catheter malfunction, and the secondary outcome was CRBSI.

In the rt-PA group, $20 \%(22 / 115)$ patients reached the primary outcome, compared with $34.8 \%(40 / 110)$ in the heparin group. CRBSI occurred in $5(4.5 \%)$ patients in the rtPA group and $15(13.0 \%)$ patients in the heparin group. The risk for adverse events was similar $(70 \%$ and $68.7 \%)$ in the rt-PA and heparin groups, respectively with no statistically significant difference in the serious adverse events between the two groups ( $20.9 \%$ and $29.6 \%$ ). Also, patients in the rtPA group did not have a higher frequency and severity of bleeding episodes [5].

This landmark study has received a lot of attention from experts in the field, as the results are very encouraging from the viewpoint of the prevention of CRBSI. The results are comparable to the outcomes reported in earlier trials involving antimicrobial locking solutions for the prevention of CRBSI among hemodialysis (HD) patients [6-12] (Fig. 1). However, the following issues merit further considerations.

The first issue is the definition of catheter malfunction. Hemodialysis catheters considered dysfunctional as per $\mathrm{K} / \mathrm{DOQI}$ guidelines if the delivered blood flow is less than $300 \mathrm{ml} / \mathrm{min}$. Currently available catheters are designed to provide blood flow of $400 \mathrm{ml} / \mathrm{min}$. The catheters are used as a bridging access until the permanent access is mature enough and the maturation time is often unpredictable. Besides the blood flow, the solute clearance needs to be included in the definition of catheter malfunction although reduced solute clearance could be the consequence of reduced blood flow. In the short-term, the formation of clots in and around the catheter, as well as fibrin sheath formation, leads to catheter dysfunction-a common complication of the HD catheter that further leads to the inability to deliver adequate dialysis. 
Catheter malfunction is a broader term and has been defined differently by different authors. Definitions include - a blood flow ranging from $200 \mathrm{~mL} /$ minute or less for a single session of dialysis, $250 \mathrm{~mL} /$ minute during two successive dialysis treatments, to less than $300 \mathrm{~mL} /$ minute for any dialysis session and alternatively, inability to initiate dialysis $[13,14]$. In the latter situation catheter replacement remains the sole option whereas in the former thrombolytic therapy may temporarily improve blood flow. Thus catheter removal appears to be a clinically more definitive marker of catheter malfunction than the peak blood flow. It appears that the optimal primary outcome obviously would have been the removal of the catheter, but the authors probably were concerned that they would not be able to enroll patients if they waited until catheters were removed due to malfunction. However, the hemodialysis guidelines actually define catheter malfunction on the basis of peak blood flow rather than the need for catheter replacement. Thus, the issue remains a matter of future debate.
The third issue is regarding the safety of rt-PA lock prophylaxis in the light of current evidence of the exit of catheter lock solutions into the circulation. Sungur et al, in their in-vitro studies performed on double lumen dialysis catheters, demonstrated a substantial amount of leak even when the corresponding catheter 'lock' volumes were used. Some catheters had lower leak ratios than the others, depending upon the size, filling volume and design [20]. The leak ratio increased significantly with $20 \%$ overfill and leakage persisted even when 'lock' volume of $20 \%$ less than the manufacturer stated catheter volume, was used. Currently, the amount of leak has been found to be clinically important in explaining the bleeding episodes reported after heparin lock placement [21]. Likewise, $0.2 \mathrm{ml}$ overfill of the stated catheter volume of gentamicin lock solution, led to plasma 'drug' concentrations close to toxic levels in an Australian study [6].

In the study of Hemmelgarn et al, the risk for adverse events was similar between the two groups; $70 \%$ of patients in the

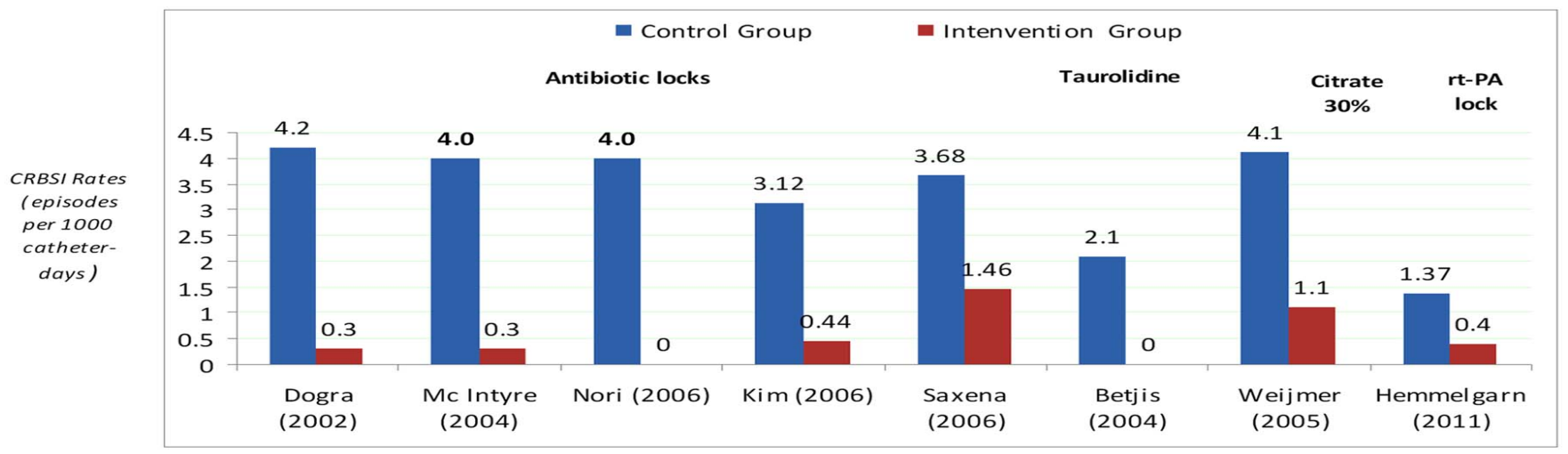

Fig. (1). Randomized controlled trials showing 60 to 100 percent reductions in hemodialysis catheter related bloodstream infections (CRBSI).

Second, Hemmelgarn et al. have inadvertently raised an interesting issue concerning the possible mechanism whereby rt-PA lock prophylaxis could cause significant reductions in CRBSIs (1.37 vs 0.40 per 1000 catheter - days, $\mathrm{P}=0.02$ ) [5]. A plausible explanation probably lies in the fact that catheter malfunction is frequently related to fibrin sheath or luminal clot formation. Fibrin, fibrinogen and fibronectin present in the thrombus enhance adherence of invading planktonic 'slime' producing bacteria, on the luminal surface of CVCs. This facilitates bacterial colonization and biofilm formation. Biofilms provide a protected environment for microorganisms for their multiplication and growth with their eventual discharge into the bloodstream causing CRBSI [3, 15, 16] (Fig. 2).

Raad et al. demonstrated a close relationship between thrombotic and infectious complications of CVCs with CRBSI rates significantly higher among patients with CVC thrombosis than in those without clot in a 16 month follow up study $(22.5 \%$ vs $0.0 \%, \mathrm{P}<0.01)$ [17]. A monthly rt-PA instillation reduced CRBSI episodes in patients with hemophilia; supporting the concept that thrombolysis could prevent CRBSI [18]. Moreover, another study demonstrated inhibition of staphylococcal bacterial growth by Urokinasetype plasminogen activator which acted as an endogenous antibiotic [19].
rt-PA group and $68.7 \%$ of patients in the heparin group had an adverse event, while reported serious adverse events were $20.9 \%$ and $29.6 \%$ in rt-PA and heparin group, respectively [5]. Likewise, patients in the rt-PA group did not have a higher frequency of bleeding episodes. However, regardless of the reported fact that there were no statistically significant differences between the two groups nearly one fifth of the patients in rt-PA group and almost one third in the heparin group, developed serious adverse events in the study with a short follow-up period of 6 months. This area warrants further long-term studies to investigate the impact of locking hemodialysis catheters with rt-PA in the light of recent reports on the exit of locking solutions in significant proportions, into the circulation - immediately after instillation and during the interdialytic period.

However, the lower likelihood of emergence of bacterial resistance could be one of the potential advantages of using rt-PA catheter lock prophylaxis. The trial sets a milestone and provides the first round findings on the new role of rtPA in the prevention of hemodialysis catheter malfunction while setting the stage for more prospective randomized trials in this field to ascertain the efficacy and address the safety issues related to rt-PA. 


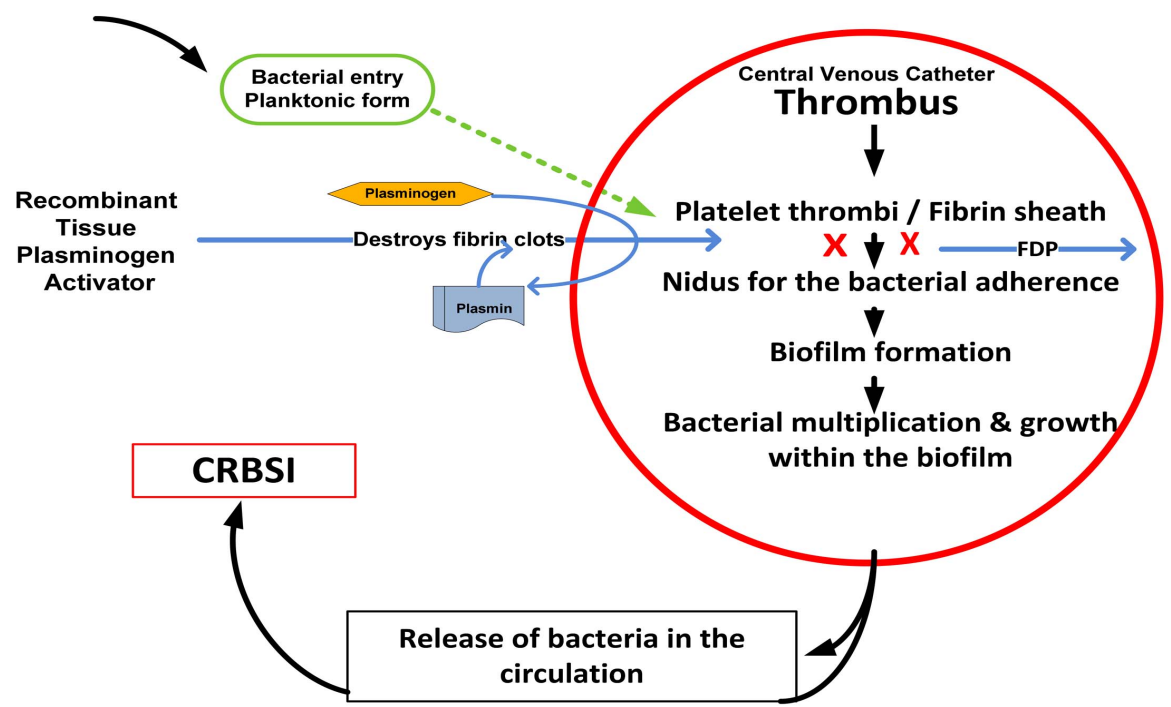

Fig. (2). Mechanism of action of tissue plasminogen activator: Tissue plasminogen activator catalyzes the conversion of plasminogen to plasmin, which then cleaves fibrin into fibrin degradation products (FDP) to dissolve the thrombus and thus prevents bacterial adherence to the catheter luminal surface.

\section{REFERENCES}

[1] Schwab SJ, Beathard G. The hemodialysis catheter conundrum: Hate living with them, but can't live without them. Kidney Int 1999; 56:1-17.

[2] US Renal Data System. USRDS 2004 Annual Data Report. Am J Kidney Dis 2005; 45:S8-S280.

[3] Saxena AK, Panhotra BR. Prevention of catheter-related bloodstream infections: an appraisal of developments in designing an infection-resistant 'dream dialysis-catheter'. Nephrology 2005; 10: $240-8$

[4] Diskin CJ, Stokes TJ, Dansby L, Radcliff L, Carter TB. Catheter locks, heparin and biofilm: what is the risk? Nephrol Dial Transplant 2008; 23(8): 2708-9.

[5] Hemmelgarn BR, Moist LM, Lok CE, et al. Prevention of dialysis catheter malfunction with recombinant tissue plasminogen activator. N Engl J Med 2011; 364: 303-12

[6] Dogra GK, Herson H, Hutchison B, et al. Prevention of tunneled hemodialysis catheter-related infections using catheter-restricted filling with gentamicin and citrate: a randomized controlled study. J Am Soc Nephrol 2002; 13: 2133-9.

[7] McIntyre CW, Hulme LJ, Taal M, Fluck RJ. Locking of tunneled hemodialysis catheters with gentamicin and heparin. Kidney Int 2004; 66: 801-5.

[8] Nori US, Manoharan A, Yee J, Besarab A. Comparison of lowdose gentamicin with minocycline as catheter lock solutions in the prevention of catheter-related bacteremia. Am J Kidney Dis 2006; 48: 596-605.

[9] Kim SH, Song KI, Chang JW, et al. Prevention of uncuffed hemodialysis catheter-related bacteremia using an antibiotic lock technique: a prospective, randomized clinical trial. Kidney Int 2006; 69: 161-4.

[10] Saxena AK, Panhotra BR, Sundaram DS, et al. Tunneled catheters' outcome optimization among diabetics on dialysis through antibiotic-lock placement. Kidney Int 2006; 70: 1629-35.
[11] Betjes MGH, van Agteren M. Prevention of dialysis catheterrelated sepsis with a citrate-taurolidine-containing lock solution. Nephrol Dial Transplant 2004; 19: 1546-51.

[12] Weijmer MC, van den Dorpel MA, Van de Ven PJ, et al. CITRATE Study Group. Randomized, clinical trial comparison of trisodium citrate $30 \%$ and heparin as catheter-locking solution in hemodialysis patients. J Am Soc Nephrol 2005; 16(9): 2769-77.

[13] Clinical Practice Guidelines for Vascular Access - Vascular Access Work Group. Am J Kidney Dis 2006; 48: S248-73.

[14] Rushing J. Caring for a patient's vascular access for hemodialysis. Nurs Manage 2010; 41(10): 47.

[15] Saxena AK. CVC: relationship between infection and thrombosis. J Vasc Access 2010; 11: S33.

[16] Mehall JR, Saltzman DA, Jackson RJ, Smith SD. Fibrin sheath enhances central venous catheter infection. Crit Care Med 2002; 30: $908-12$.

[17] Raad II, Luna M, Khalil SA, et al. The relationship between the thrombotic and infectious complications of central venous catheters. JAMA 1994; 271: 1014-6.

[18] Weck S, Cheung S, Hiraoka-Sutow M, Patapoff T, Semba CP Alteplase as a catheter locking solution: in vitro evaluation of biochemical stability and antimicrobial properties. J Vasc Interv Radiol 2005; 16 (3): 379-83.

[19] Jin T, Bokarewa M, Tarkowski A. Urokinase-type plasminogen activator, an endogenous antibiotic. J Infect Dis 2005; 192(3): 42937.

[20] Sungur M, Eryuksel E, Yavas S, Bihorac A, Layon AJ, Caruso L. Exit of catheter lock solutions from double lumen acute hemodialysis catheters--an in vitro study. Nephrol Dial Transplant 2007; 22(12): 3533-7.

[21] Polaschegg HD, Shah C. Overspill of catheter locking solution safety and efficacy aspects. ASAIO J 2003; 49: 713-5. 